\title{
Predator-prey interactions cause apparent competition between marine zooplankton groups
}

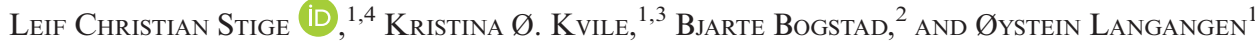 \\ ${ }^{1}$ Centre for Ecological and Evolutionary Synthesis (CEES), Department of Biosciences, University of Oslo, \\ P.O. Box 1066, N-0316 Oslo, Norway \\ ${ }^{2}$ Institute of Marine Research, P.O. Box 1870 Nordnes, N-5817 Bergen, Norway
}

\begin{abstract}
Predator-mediated apparent competition is an indirect negative interaction between two prey species mediated by a shared predator. Quantifying such indirect ecosystem effects is methodologically challenging but important for understanding ecosystem functioning. Still, there are few examples of apparent competition from pelagic marine environments. Using state-space statistical modeling, we here provide evidence for apparent competition between two dominant zooplankton groups in a large marine ecosystem, i.e., krill and copepods in the Barents Sea. This effect is mediated by a positive association between krill biomass and survival of the main planktivorous fish in the Barents Sea, capelin Mallotus villosus, and a negative association between capelin and copepod biomasses. The biomass of Atlantic krill species is expected to increase in the Barents Sea due to ongoing climate change, thereby potentially negatively affecting copepods through apparent competition. By demonstrating and quantifying apparent competition in a large marine ecosystem, our study paves the way for more realistic projections of indirect ecosystem effects of climate change and harvesting.
\end{abstract}

Key words: apparent competition; indirect ecosystem effects; large marine ecosystem; predator-prey dynamics; state-space model; zooplankton-fish interactions.

\section{INTRODUCTION}

The term "apparent competition" was introduced by Robert D. Holt in 1977 to describe an indirect, negative interaction between two species mediated by a third species other than their prey (Holt 1977). We here focus on predator-mediated apparent competition, whereby a species or species group negatively affects another species or species group that share the same predator by influencing predator abundance or biomass. Some empirical evidence of apparent competition exists, indicating that such effects may be widespread in nature (Chaneton and Bonsall 2000, DeCesare et al. 2010). For example, in marine temperate reefs, predatory rockfish mediate apparent competition between shrimp groups (Frid and Marliave 2010). However, apparent competition may be difficult to detect empirically due to its indirect nature and the potential for concurrent exploitative competition or other community effects (DeCesare et al. 2010), and is less well studied than other indirect effects such as exploitative competition (Wooton 1994). A good understanding of both direct and indirect effects in ecological communities is fundamental for anticipating how ecosystems respond to natural and human influences. For example, climate

Manuscript received 28 September 2017; revised 17 November 2017; accepted 8 December 2107. Corresponding Editor: Steven G. Morgan.

${ }^{3}$ Current address: Department of Biology, Woods Hole Oceanographic Institution, Woods Hole, Massachusetts, 02543 USA.

${ }^{4}$ E-mail: 1.c.stige@ibv.uio.no impacts on natural populations are more likely to be mediated indirectly through altered species interactions than through direct, abiotic effects (Ockendon et al. 2014). Similarly, accounting for species interactions is important for sustainably managing harvested populations, as advocated in the ecosystem approach to fisheries management (May et al. 1979, Botsford et al. 1997, Pikitch et al. 2004).

We here analyze predator-prey dynamics between a key pelagic fish species and two dominant zooplankton groups in a large marine ecosystem (Fig. 1). Specifically, we focus on capelin, which is the main predator on zooplankton in the Barents Sea (Dalpadado et al. 2012), and copepods and krill, which together constitute about $80 \%$ of its diet (Dalpadado and Mowbray 2013). During years with high capelin biomass, krill and copepod biomasses are significantly reduced in the capelin's main feeding areas in the central and northern Barents Sea, suggesting strong top-down effects (Skjoldal et al. 1992, Dalpadado et al. 2002, Eriksen and Dalpadado 2011, Stige et al. 2014, Langangen et al. 2017). Bottom-up effects by zooplankton on capelin are suggested by positive correlations of zooplankton biomass with individual growth of capelin (Gjøsæter et al. 2002), while associations with survival are to our knowledge unknown. We here use time series covering more than 3 decades on zooplankton biomass, capelin length and abundance at age and landings in order to estimate top-down effects by capelin on zooplankton and bottom-up effects by zooplankton on growth, survival and reproduction of capelin (Fig. 1). Specifically, we develop a statistical model of intermediate 

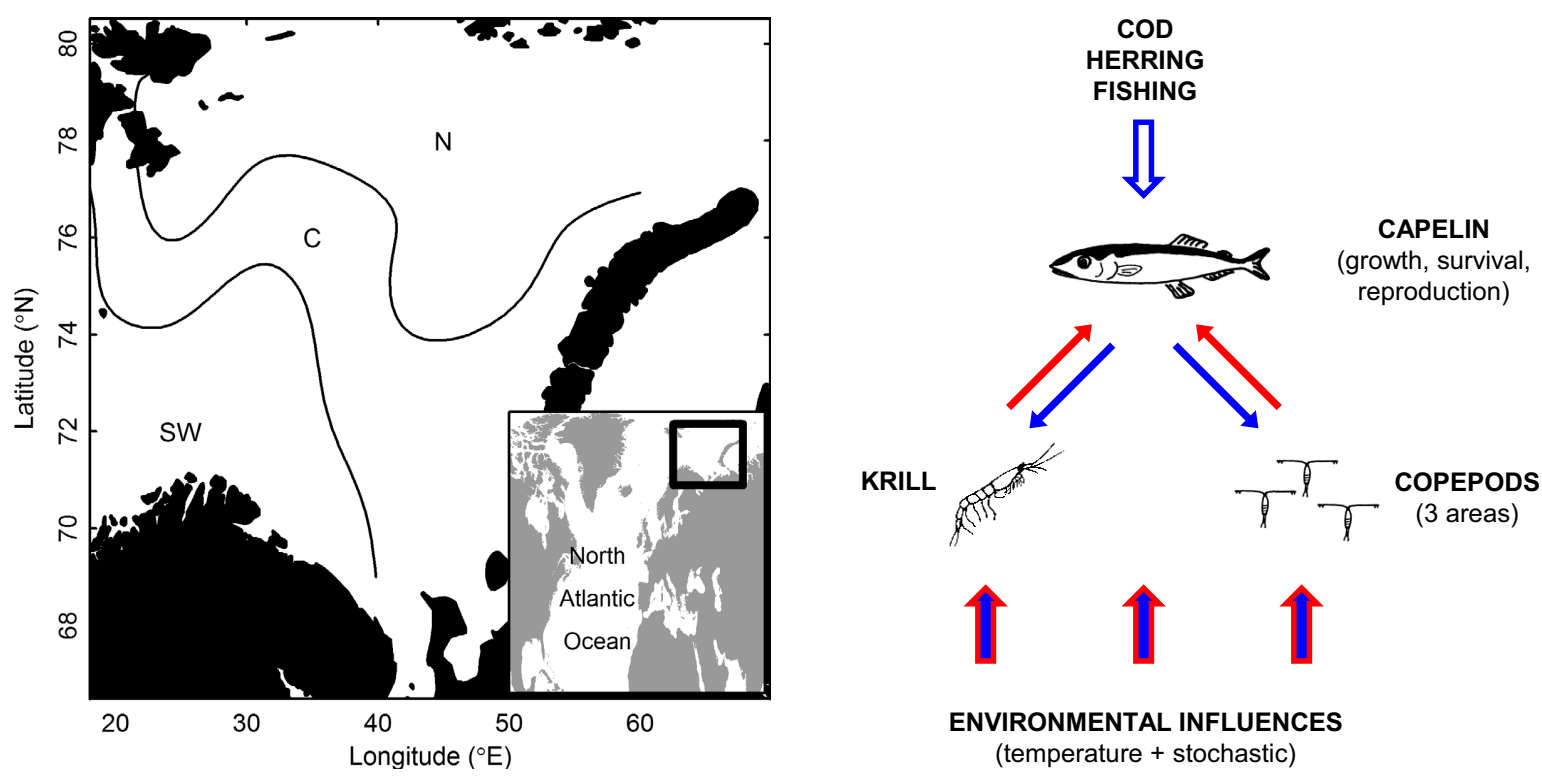

FIG. 1. The Barents Sea study system. The map shows the location of the Barents Sea and the division into three regions based on mean sea temperature $(T)$ at $100 \mathrm{~m}$ depth during copepod sampling in August-September 1981-2010. SW, southwestern $\left(T>3^{\circ} \mathrm{C}\right)$. C, central $\left(T 0-3^{\circ} \mathrm{C}\right)$. N, northern $\left(T<0^{\circ} \mathrm{C}\right)$. The diagram to the right illustrates the modeled two-way interactions among copepods, krill and capelin (narrow arrows) and the effects of external variables (broad arrows). Bottom-up effects are shown in red, top-down in blue.

complexity, with the model parameters for bottom-up and top-down effects estimated jointly from the data. Hence we aim to draw empirically based inferences while minimizing the risk of model bias and spurious results (Plagányi et al. 2014, Collie et al. 2016). We use the statistical model results to assess implications of zooplanktoncapelin interactions for population and multi-species dynamics, allowing us to detect apparent competition between krill and copepods.

\section{Material ANd Methods}

\section{General outline of analysis}

The purpose of the analysis was to estimate effects of zooplankton on population dynamics of capelin while assessing feedback-effects from capelin on zooplankton. To do so, we used a Bayesian state-space approach, whereby effects both ways, by zooplankton on capelin and by capelin on zooplankton, were described by a process model (shown schematically in Fig. 1 and more detailed in Fig. S1: Appendix S1). By simultaneously estimating parameters for bottom-up and top-down effects we find the parameter values that best describe the dynamics as a whole. We also account for effects of temperature, fish predators and fisheries. Furthermore, we structure the statistical model so that it captures the linkages between growth, reproduction and spawning mortality implied by the life history of Barents Sea capelin, which is thought to have length-dependent maturation and semelparous reproduction (Gjøsæter 1998). The process model was linked to data by an observation model. This way, uncertainties about biological processes and observation noise were explicitly accounted for, to provide unbiased parameter estimates and appropriate confidence bands (Clark and Bjørnstad 2004).

\section{Data}

The capelin data analyzed were abundance-at-age 0-4 (millions) and length-at-age $0-4(\mathrm{~cm})$ in autumn and total reported catches (tonnes) in summer-autumn and in winter fisheries. The capelin series analyzed covered the period 1980-2015. Age-0 fish were sampled by pelagic 0-group trawl surveys in August-September and older age-classes by acoustic surveys in SeptemberOctober carried out by the Institute of Marine Research (IMR), Bergen, Norway, and the Polar Research Institute of Marine Fisheries and Oceanography (PINRO), Murmansk, Russia. Estimates of absolute abundance directly based on these surveys were taken from ICES reports (2016), which also provided catch data. Length data were provided by IMR and annual IMR-PINRO survey reports.

We analyzed biomass area density ( $\mathrm{g}$ dry weight $\mathrm{m}^{-2}$ ) of mesozooplankton, for brevity referred to as "copepods" due to their dominance in the mesozooplankton biomass (Orlova et al. 2011), in three areas of the Barents Sea. The three areas were the southwestern (characterized by surface waters $\left.>3^{\circ} \mathrm{C}\right)$, central $\left(0-3^{\circ} \mathrm{C}\right)$ and northern $\left(<0^{\circ} \mathrm{C}\right)$ Barents Sea (following Stige et al. 2014, with temperatures referring to climatologies for the time 
of the zooplankton surveys). Biomass time-series were constructed as in Dalpadado et al. (2012) and Stige et al. (2014), updated to 2015 by use of more recent spatiotemporal zooplankton data from the IMR data base. Copepods were sampled from August to early October 1981 and 1984-2015 from close to the bottom to surface by IMR. The majority of the samples were taken using WP2 plankton nets (56 cm diameter, $180 \mu \mathrm{m}$ mesh). The dominant species represented by the indices are Calanus finmarchicus in the southwestern part and Calanus glacialis and Calanus hyperboreus in central and northern parts (Orlova et al. 2011).

An index of krill biomass area density in the entire Barents Sea was constructed by taking the arithmetic mean of day and night catches $\left(\mathrm{g} / \mathrm{m}^{2}\right)$ from pelagic trawls in August-October 1980-2015 (Eriksen et al. 2016a). The dominant species represented by the index are Thysanoessa inermis, Thysanoessa raschii and Meganyctiphanes norvegica (Eriksen et al. 2016b). Whereas we only had area-aggregated data for krill, we assessed the influence of aggregating zooplankton data by comparing analyzes of area-resolved and area-aggregated copepod indices (see Sensitivity analysis in Appendix S1).

Monthly sea surface temperature for $68-80^{\circ} \mathrm{N}$ and $20-50^{\circ} \mathrm{E}$ at a $2 \times 2^{\circ}$ grid (NOAA_ERSST_V3 data set) were provided by the NOAA/OAR/ESRL PSD, Boulder, Colorado, USA, from their Web site at http://www.esrl. noaa.gov/psd/.

Annual biomasses of key predators on capelin, i.e., age 3-6 cod and age 1-2 herring (Hjermann et al. 2004, 2010), were obtained from ICES (2016). The estimates were from virtual population analyzes, mostly relying on fisheries catch data. Other potential predators, such as marine mammals (Bogstad et al. 2015), were not explicitly modelled due to scarcer observation data, but were accounted for in a process noise term in the model.

\section{Statistical model}

A detailed description of the statistical model is given in Appendix S1. The model quantified effects of copepods and krill on capelin length and abundance at ages 0 to 4 years and effects of capelin biomass on biomasses of copepods and krill. Age-0 capelin were assumed to interact with copepods in the southwestern Barents Sea, age- 1 capelin with copepods in the central Barents Sea and age- 2 and older capelin with copepods in the central and northern Barents Sea (Stige et al. 2014). Capelin biomass was calculated from the modelled abundanceat-age and length-at-age based on length-weight relationships described in the literature (Monstad 1971, Gjøsæter 1998). Maturation depends on length with $50 \%$ maturity around length $L_{p 50}=14 \mathrm{~cm}$ (Gjøsæter 1998, Baulier et al. 2012). Hence, variable fractions of 2- and 3-year-olds contribute to the following year's spawning stock, depending on the mean length of the year-class. We assumed that all spawning capelin that have spawned die shortly after spawning (Gjøsæter
1998). The model also quantified effects of temperature on capelin lengths and age- 0 abundance, and of cod, herring and commercial catches on capelin abundances, with the choice of predictor variables based on previous literature (Gjøsæter and Loeng 1987, Skjoldal et al. 1992, Gjøsæter and Bogstad 1998, Dalpadado et al. 2002, 2012, Gjøsæter et al. 2002, Hjermann et al. 2004, 2010, Pedersen and Fossheim 2008, Orlova et al. 2009, 2010, Eriksen and Dalpadado 2011, Stige et al. 2014), as detailed in the Supplementary Methods in Appendix S1. The parameters estimated by the model are listed in Table S1 and the variables in Table S2: Appendix S1.

The following generic equation describes the dynamics of capelin abundance:

$$
\begin{aligned}
x_{A, T}= & \mathrm{nA} 0+x_{A-1, T-1}-F_{A-1, T-1}-M_{A-1, T-1} \\
& +\mathbf{n}_{\mathbf{A}} \boldsymbol{E}_{\boldsymbol{T}-\boldsymbol{1}}+e_{A, T}
\end{aligned}
$$

Here, $x_{A}, T$ is capelin log-abundance at age $A$ (for $A \geq 1$ ) in year $T, \mathrm{nA} 0$ is an age-specific intercept, $F_{A}, T$ is fishing mortality rate (which also enters into catch equations), $M_{A}, T$ is maturation rate (a function of the modelled mean length of the age-class), $\mathbf{n}_{\mathbf{A}}$ is a row vector of coefficients for effects of environmental variables, $\boldsymbol{E}_{\boldsymbol{T}}$ is a column vector with environmental values for year $T$ (i.e., temperature and log-biomasses of potential predators and zooplankton prey groups), and $e$ is the process noise, which captures effects of predictor variables not explicitly modelled. This formulation assumes additive effects of environmental variables on mortality rate and multiplicative effects on abundance, which is not true if, for example, predator effects on capelin mortality depend on capelin abundance (Hjermann et al. 2004). As part of the sensitivity analysis (Appendix S1) we assessed how this assumption influenced results. To quantify bottom-up effects by copepods on capelin, modelled copepod biomasses in the regions occupied by each capelin age group were averaged. Density dependence in survival to ages $\mathrm{A}>0$ was assumed to be mediated through zooplankton biomass only, while a Gompertz model (Ives et al. 2003) was used to link maturing stock biomass to recruitment to age $A=0$.

Analogously, capelin log-length $y$ was modelled as:

$$
y_{A, T}=110+111 \hat{y}_{A-1, T-1}+\mathbf{l}_{\mathbf{A}} \boldsymbol{E}_{\boldsymbol{T - 1}}+d_{A, T}
$$

Here, $y$ is log-length of the immature fraction of the year-class (as maturing capelin were assumed to die shortly after spawning), 110 and 111 are parameters for a Gompertz type growth function, $\mathbf{l}_{\mathbf{A}}$ are coefficients for environmental effects, and $d$ is process noise.

Log-biomasses of krill and copepods were modelled using Gompertz models that included top-down effects of capelin biomass (sum of ages potentially preying on the given zooplankton group) and process noise. The baseline model included no direct interaction between copepods and krill, but as part of the sensitivity analysis (Appendix S1) we included parameters for exploitative 
competition between the two zooplankton groups. Copepod biomasses in the three Barents Sea regions were described by separate process equations.

The state variables for capelin abundances, capelin lengths, capelin catches, copepod biomasses and krill biomass were linked to observation data by an observation model. For example, observed log-abundance $\hat{x}$ of capelin was a function of the modelled log-abundance $x$ and observation noise $\varepsilon$ :

$$
\hat{x}_{A, T}=x_{A, T}+\varepsilon_{A, T}
$$

Note that while time-series for both age- 3 and age- 4 capelin were included in the analysis, we assumed that predictor effects on these age classes were the same and hence described by the same parameters. This simplification was motivated by the overlapping size-range and hence similar ecology of these age-classes (e.g., Fig. 2), and the general low abundance at age 4 , which we assumed led to elevated observation error.

\section{Model estimation}

We used a Bayesian Markov Chain Monte Carlo (MCMC) approach to jointly estimate all parameters in
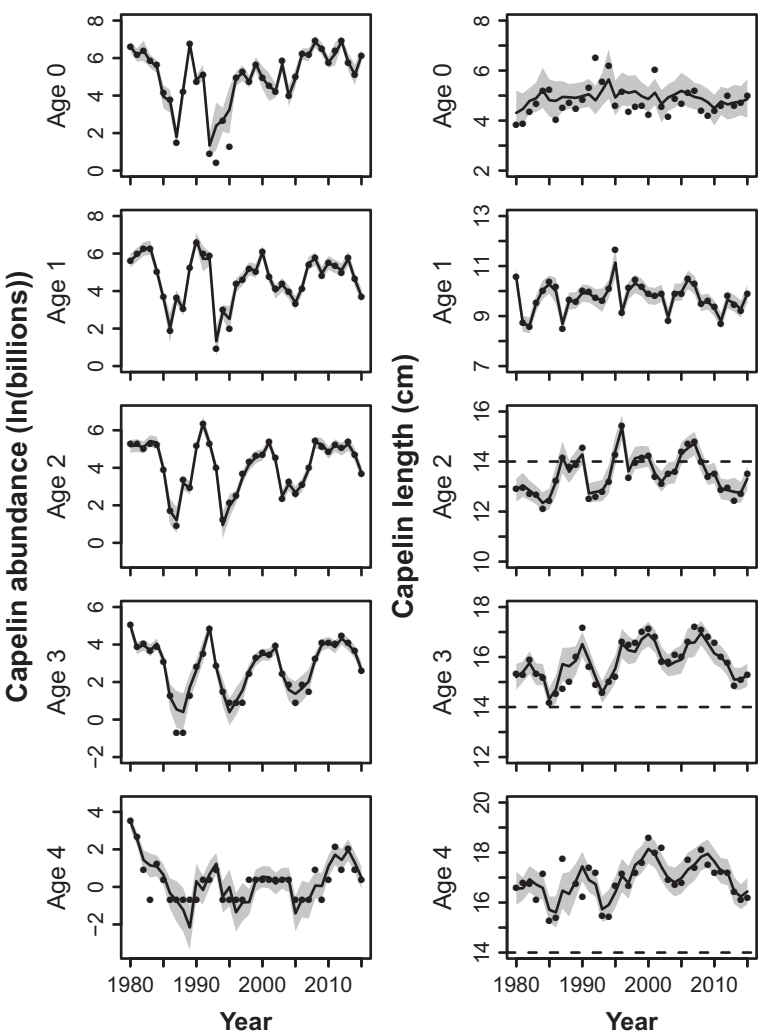

FIG. 2. Modeled and observed time-series. Lines and grey shading show the median and $95 \%$ credibility intervals from posterior distributions of latent variables for capelin abundance-at-age, capelin length-at-age, capelin biomass and zooplankton biomass. Points show observation data. All observation data except capelin stock biomasses were used to fit the model. Broken lines in panels for capelin length show length at maturation $\left(L_{p 50}\right)$. the model. For this purpose, we used the program JAGS (Just Another Gibbs Sampler) and the rjags and dclone packages of R (Solymos 2010, Plummer 2016). The likelihood function was created based on the model and the data, and in combination with the prior distributions of the parameters, the posterior distributions were estimated. Prior distributions for the parameters in the process model were uniform and broad in order to let the data drive the inferences. We used three independent chains with 1, 300, 000 iterations, where the first 300, 000 iterations were used as 'burn-in' iterations to ensure that the chains had converged. In addition, we thinned the chains with a factor 1,000 to reduce autocorrelation in the posterior samples and to produce a reasonable amount of output, in this case resulting in 1,000 samples from each chain, in total 3,000. We used the Gelman and Rubin $\hat{R}$ convergence diagnostics (Gelman and Rubin 1992) and visual inspection of the chains to ensure convergence.

\section{Assessing food-web effects of changes in krill or copepod biomass}

To assess some of the implications of the findings for our understanding of zooplankton-capelin dynamics, we
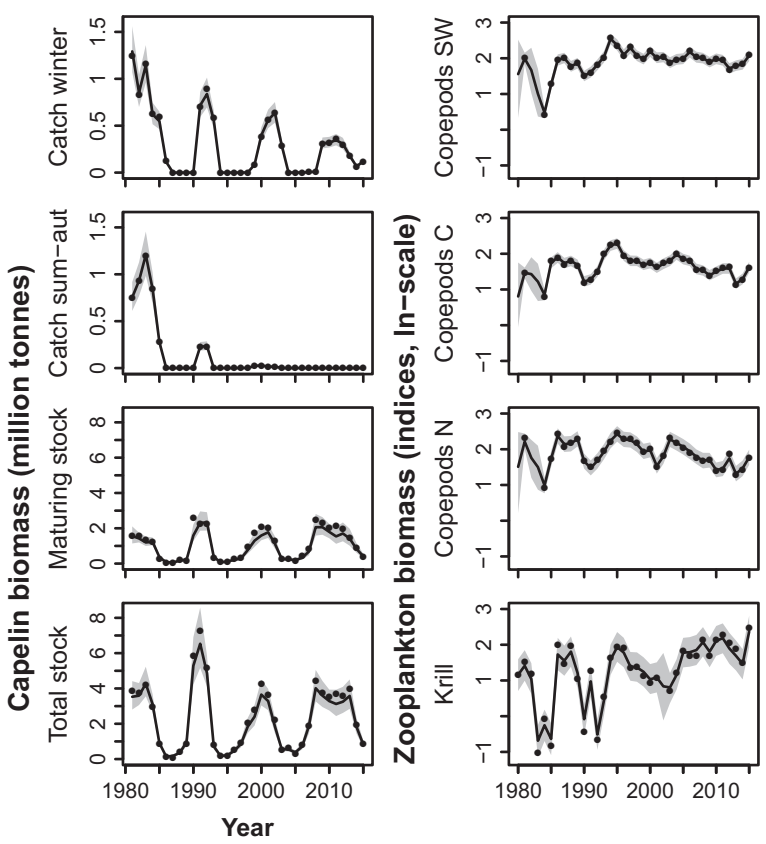

Year 
perturbed the model by increasing krill or copepod biomass and compared population trajectories with the non-perturbed situation. We considered both pulse perturbations, where copepod or krill biomass was increased by 1 on the natural log-scale for a single year, and sustained perturbations, where the overall productivity of copepods or krill, represented by the intercept in the log-scale Gompertz models, was increased by 0.1 . For the different perturbations we assumed that other conditions remained unaffected, by drawing from the posterior distribution as described in Langangen et al. (2017). Pulse perturbations were iteratively applied to all years and differences between non-perturbed and perturbed biomasses at different time-lags after the perturbation were recorded. Sustained perturbations were applied from the second year onwards and differences between nonperturbed and perturbed biomasses were recorded.

The data sets and computer code supporting this article have been uploaded as part of the supplementary material.

\section{RESULTS}

The model captured the variability in the observed time-series well, with no systematic deviations between fitted and observed values suggestive of model misspecification (Fig. 2). Further model diagnostics and sensitivity analyzes suggested that the main findings were robust to key assumptions of the model (Supplementary Results in Appendix S1).

The analysis supported negative top-down effects by capelin on copepods and krill, as shown by predominantly negative posterior distributions for the model parameters quantifying these effects (Fig. 3a). The 95\% credibility intervals excluded zero for capelin effects on copepods in the central and northern Barents Sea, providing strong evidence for negative effects, but overlapped with zero for effects on krill and on copepods in the southwestern Barents Sea.

The analysis further suggested significant bottom-up effects of copepods and krill on survival and growth of capelin (Fig. 3b-e). Krill biomass associated most strongly and positively with survival, i.e. with year-toyear changes in abundance of capelin year-classes (Fig. 3d). A doubling in krill biomass is, for example, predicted to lead to around $40 \%$ higher capelin survival to ages 2, 3 and 4 years, which translate into the same percentage increases in biomass-at-age (due to the linear relationship between abundance and biomass), and to lead to around $1-2 \%$ increases in capelin length at the same ages, which in turn translate into around 3-5\% increases in biomass-at-age (due to the approximately cubic relationship between length and biomass). Results thus show that krill effects on capelin biomass operate mainly through survival, with effects through growth being an order of magnitude weaker. Note also that krill a. Capelin effect on zooplankton biomass

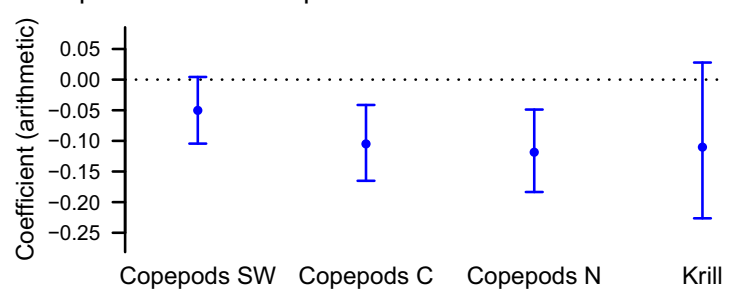

b. Copepod effect on capelin abundance at age

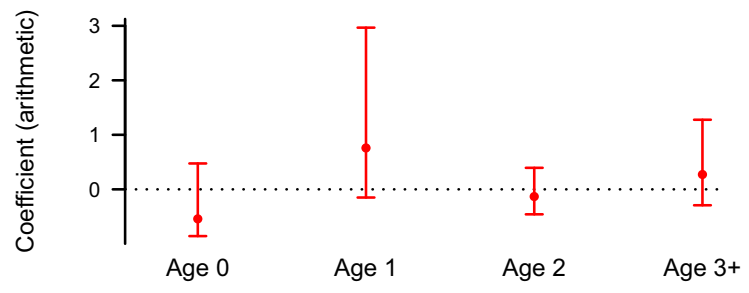

c. Copepod effect on capelin length at age

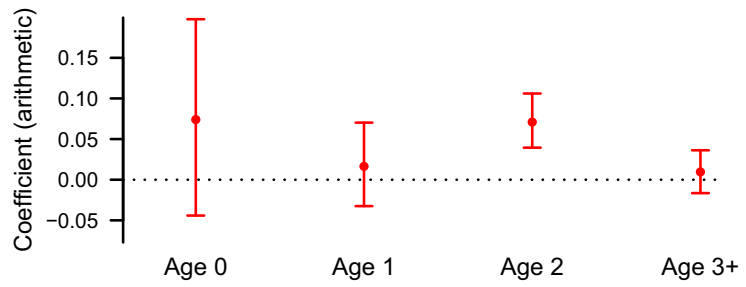

d. Krill effect on capelin abundance at age

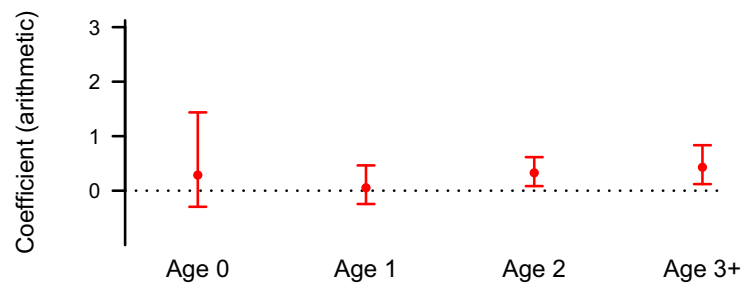

e. Krill effect on capelin length at age

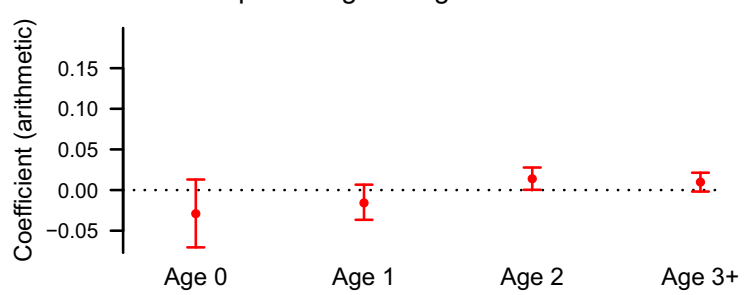

FIG. 3. Parameter estimates for zooplankton-capelin interactions. The coefficients are transformed to arithmetic scale and show the proportional change in the response (i.e., zooplankton biomass, capelin abundance or capelin length) per doubling in the predictor (i.e., capelin or zooplankton biomass). See Table S3: Appendix S1 for untransformed values for all model parameters. Filled circles: marginal posterior means. Solid lines: 95\% credibility intervals. Dotted lines: zero line. Bottom-up effects are shown in red, top-down in blue. Zooplankton effects on age- 3 and age- 4 capelin are represented by the same parameters ("Age 3+"). 
biomass varied more among years than copepod biomass, with the among-year standard deviation on logscale being more than twice as high, which together with the estimated coefficient values imply stronger bottomup effects of the observed krill fluctuations than the observed copepod fluctuations.

Copepod biomass associated positively with growth, i.e. with year-to-year changes in mean length of capelin year-classes, especially to age 2 (Fig. 3c). A doubling in copepod biomass in the central and northern Barents Sea is predicted to lead to around $7 \%$ increase in length of 2-year-old capelin, which translates into around $25 \%$ increase in biomass of this age group. Note that the estimated positive effects on length at age translate into positive effects on maturation at age, as maturation was a function of length. The estimates for effects of copepod biomass on capelin survival were uncertain with credibility intervals overlapping with zero for all ages (Fig. 3b).

The analysis further showed positive associations of temperature with capelin age- 0 abundance and age- 2 length, negative association of juvenile herring with capelin age- 0 abundance, and negative associations of cod with capelin abundance at most ages. The marginal posterior distributions of all model parameters are shown in Figs. S2-S4 and Table S3: Appendix S1.

By perturbing the model we found that a 1-yr increase in krill biomass ( +1 on $\ln$-scale) leads to increased capelin biomass for approximately $4 \mathrm{yr}$ and significantly lower copepod biomass in central and northern areas compared to the biomass trajectories without such a krill increase (Fig. 4). The consequences of a corresponding 1-yr increase in copepod biomass were more uncertain, with the $95 \%$ credibility intervals for the indirect effect on krill encompassing both positive and negative effects. Our results also indicate that copepods have more transient effects on capelin biomass than krill do (Fig. 4), as positive copepod effects on growth translate into negative effects on abundance at ages 3 and 4 after 2-3 years because of spawning mortality (Figs. S5-S6: Appendix S1). Sustained perturbations, where the overall productivity of krill or copepods is increased, have corresponding long-term effects, with krill negatively affecting copepods but with more uncertain effects the other way. The direct and indirect effects are of comparable orders of magnitude: we find that if krill productivity increases with $11 \%(+0.1$ in the intercept in an ln-scale Gompertz model), krill biomass increases with around $14 \%$, capelin biomass with around $18 \%$, and copepod biomass in the central and northern Barents Sea decreases with around $4 \%$, but with $95 \%$ credibility intervals being wide and only partly excluding zero.

\section{Discussion}

We here demonstrate apparent competition in a large marine ecosystem. Apparent competition, implying a succession of bottom-up and top-down effects, has long been recognized as a potentially widespread ecological phenomenon (Holt 1977). However, few examples of apparent competition exist from pelagic marine systems, despite many suggestions of strong top-down effects (Frank et al. 2005, Casini et al. 2009, Boyce et al. 2015), also in combination with bottom-up effects (Lynam et al. 2017). Indirect interactions can be more difficult to detect than direct predator-prey interactions, but be pivotal for our understanding of how ecosystems will respond to changes in climate, harvesting or other external factors.

Using state-space statistical modeling, we have elucidated the dynamics between a dominant planktivorous fish and its zooplankton prey, finding that top-down, bottom-up and indirect effects come into play. Our model also accounts for temperature effects as well as herring and cod predatory effects, with the estimated coefficients generally consistent with previous studies on capelin growth (Gjøsæter and Loeng 1987) and population dynamics (Hjermann et al. 2004, 2010).

The estimated top-down effects are consistent with previous studies showing inverse correlations of capelin with copepod and krill time-series (Skjoldal et al. 1992, Dalpadado et al. 2002, Eriksen and Dalpadado 2011, Stige et al. 2014, Langangen et al. 2017). Capelin schools appear to be able to forage down the zooplankton biomass in an area in a matter of 3-4 d (Hassel et al. 1991) and the capelin form density-dependent migratory waves that move out from the central distribution area as the zooplankton prey are depleted locally (Fauchald et al. 2006). The finding that top-down effects on copepods are stronger in central and northern than in southwestern Barents Sea is consistent with previous analyzes (Stige et al. 2014) and with the location of the main feeding areas of capelin (Gjøsæter 1998, Eriksen et al. 2017).

Positive bottom-up effects of copepod and krill biomass on capelin growth are supported by studies in which individual growth was estimated from otoliths (Gjøsæter et al. 2002). We find strongest statistical support for copepod effects on capelin at age 2 years. Weak copepod effect on capelin at age $3+$ is consistent with a gradual shift in capelin diet with age from dominance of copepods to krill (e.g., Gjøsæter and Bogstad 1998, Orlova et al. 2010). Uncertain estimates of copepod effects on capelin at ages 0 and 1 are likely caused by uncertain capelin lengths at age 0 (Fig. 2). A relatively weak association between capelin growth and krill biomass variations is possibly related to the bulk of the krill biomass being south of the main summer feeding areas of capelin (Eriksen et al. 2017), which may also explain why estimates of top-down effects by capelin on krill are uncertain. Negative capelinkrill associations have previously been found to be strongest and most consistent over time north of $74{ }^{\circ} \mathrm{N}$ in the Barents Sea, i.e., the part of the krill distribution that overlaps most with the main feeding areas of adult capelin (Eriksen and Dalpadado 2011).

Our results suggest that capelin survival is positively associated with krill, while the association with copepods is more uncertain. We interpret this finding in the 
Pulse perturbation

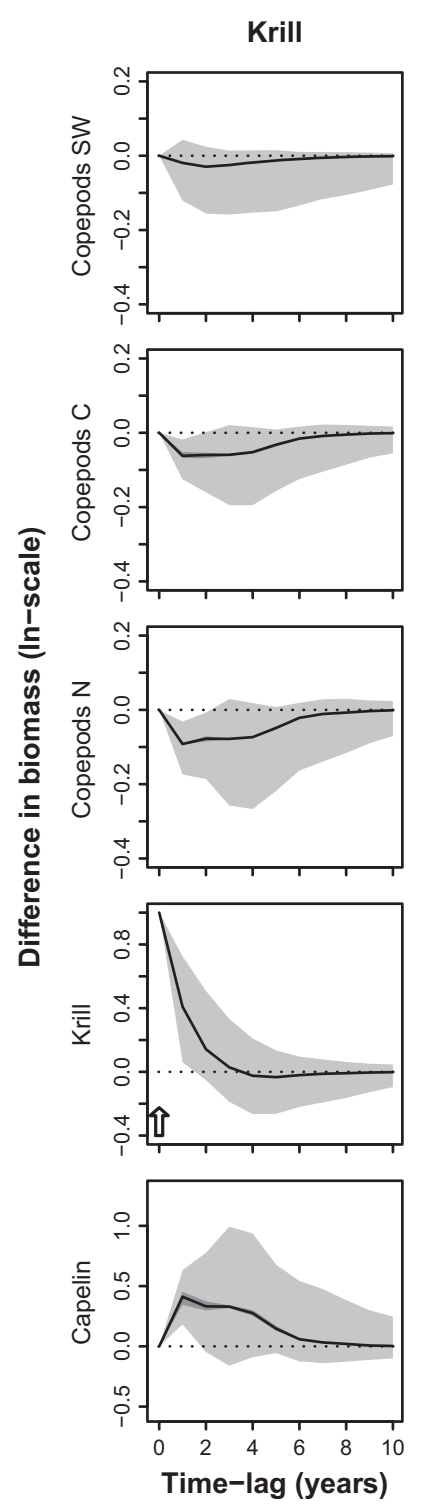

\section{Copepods}
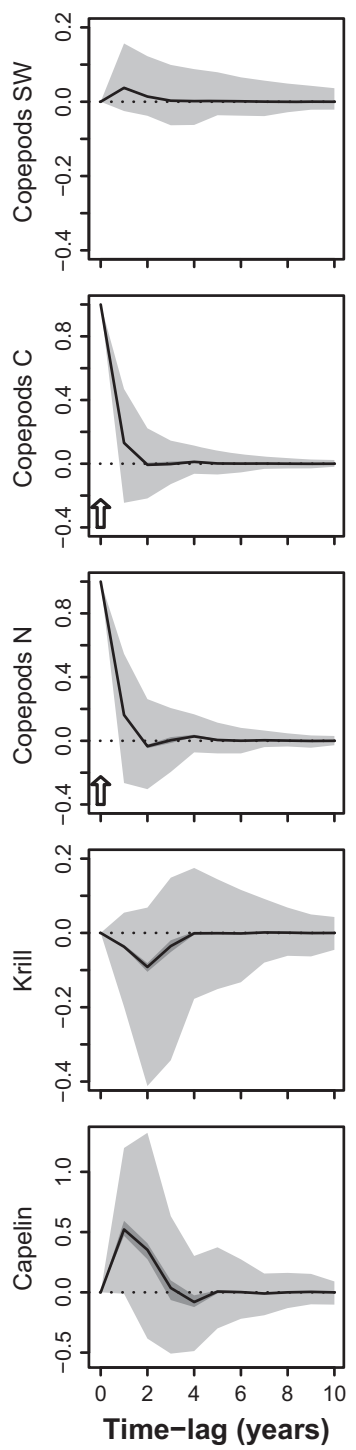

Sustained perturbation
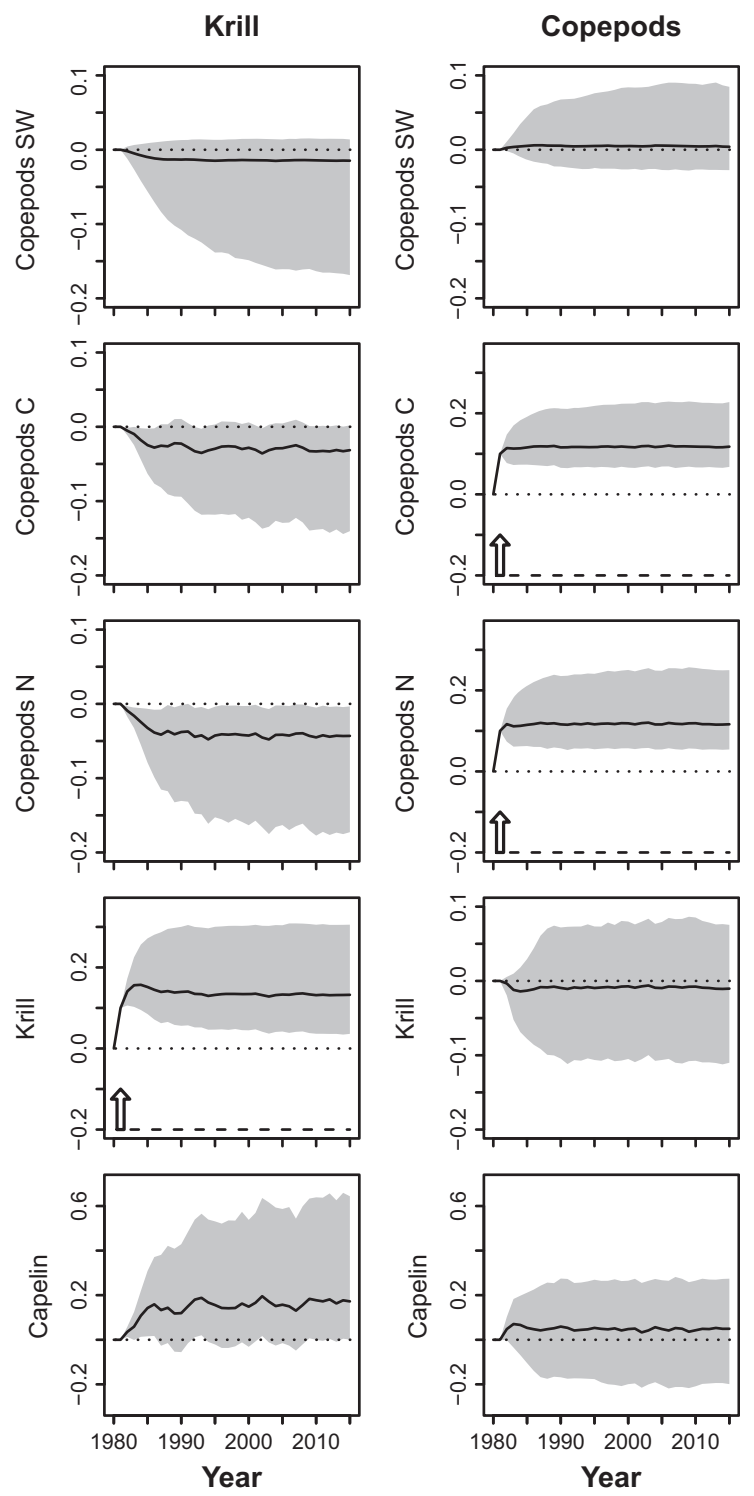

FIG. 4. Food web effects of increase in krill or copepod biomass. Each column represents either a pulse perturbation or a sustained perturbation for one zooplankton group (represented by arrows and, for sustained perturbations, broken lines). Pulse perturbation: biomass is increased by 1 unit on $\ln$-scale for $1 \mathrm{yr}$. Sustained perturbation: the overall productivity (the intercept in a Gompertz model) is increased by 0.1 on $\ln$-scale. Unbroken lines: median predicted difference between perturbed and unperturbed ln-biomass. Dark grey bands for pulse perturbations show interannual variability, i.e., interquartile range in median predicted difference depending on impact year (most evident for pulse perturbation effects on capelin). Grey bands: $95 \%$ credibility intervals (median across impact years for pulse perturbations). Stippled lines: zero effect. See Figs. S5, S6: Appendix S1 for full versions of these figures.

light of the seasonal feeding cycle of capelin. Long-term data show that capelin hardly eat during winter, from November to January (Ushakov and Prozorkevich 2002). Then, in February, capelin first start feeding on krill in the southwestern parts of the Barents Sea (Gjøsæter 1998, Ushakov and Prozorkevich 2002). The main feeding season is summer and autumn, from July to October, when capelin follow the retreating ice edge northwards while feeding on high concentrations of lipid-rich copepods and krill (Gjøsæter 1998, Ushakov and Prozorkevich 2002, Dalpadado and Mowbray 2013). Buren et al. (2014) suggest that food conditions for capelin in spring, when the stored energy level is low, might influence starvation or predation mortality and mediate bottom-up climate effects on capelin biomass in the marine ecosystem off the Newfoundland and Labrador Shelf (but see Frank et al. 2006 for an alternative interpretation). We hence speculate that the krill-capelin 
survival association in the Barents Sea is caused by food limitation in spring causing starvation or increased susceptibility to predation.

By jointly estimating bottom-up and top-down effects in a process based model we can infer implications of predator-prey interactions for multi-species dynamics. To illustrate these implications, we provide the following scenario for the food web effects of a temporary increase in krill biomass, for example caused by good krill recruitment (Fig. 5). Partly because of compensatory density dependence among the krill (zk1 parameter, Fig. S4: Appendix S1), this "boost" in krill biomass gradually subsides over time, nonetheless providing improved feeding conditions for capelin for several years. Capelin biomass thus increases, mainly through a positive association between krill biomass and capelin survival. The high capelin biomass provides a negative feedback effect on the krill, but this effect is not strong enough to significantly overcompensate for the previous increase, which could otherwise have led to predator-prey cycles (May 1974). On the other hand, the high capelin biomass leads to increased predation and reduced biomass of copepods in years following the boost in krill biomass. The result is apparent competition between krill and copepods in the Barents Sea. A delay from the increase in krill to the reduction in copepod biomass is consistent with our interpretation that a large overwintering population of krill leads to high capelin survival in spring and subsequently high consumption through the main feeding season of capelin in summer. Whether the apparent competition is asymmetric, with only krill adversely affecting copepods and not vice versa, remains undecided. Post hoc regression analyzes of copepod and krill time-series support a delayed negative association going from krill to copepods but not from copepods to krill, which shows that also simpler analysis methods support quantitatively important krill effects on copepod dynamics (Table S4: Appendix S1).

The dominant copepod and krill species in the Barents Sea have similar herbivorous diets (Dalpadado et al. 2008, Søreide et al. 2010), but it appears unlikely that the negative association between copepod and krill biomasses is caused by exploitative competition. Firstly, sensitivity

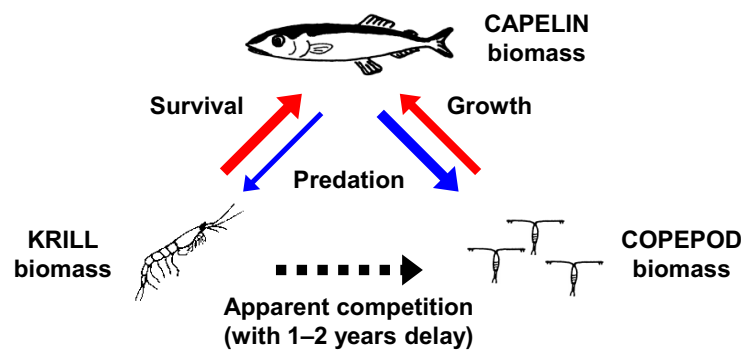

FIG. 5. Schematic presentation of the finding of apparent competition between krill and copepods in the Barents Sea. Bottom-up effects are shown in red, top-down in blue. Arrow widths are approximately proportional to estimated effect sizes (standardized by dividing by standard errors). analysis shows that direct interactions between copepods and krill are statistically non-significant in a model that also allows for indirect effects through capelin, and that accounting for direct interactions has little influence on parameter estimates for the indirect effects (Appendix S1). Secondly, previous studies suggest that fish predation explains $>50 \%$ of the year-to-year variation in copepod biomass concentration in the central and northern Barents Sea (e.g., Stige et al. 2014), whereas statistical support for significant bottom-up effects by phytoplankton on copepod biomass in these areas appears to be weaker (Dalpadado et al. 2014). Hence, in our view, indirect effects of krill on copepods through predators, i.e. apparent competition, appear more parsimonious than indirect effects through prey, i.e. exploitative competition. Strong predator-prey coupling among capelin and zooplankton is possibly related to the dominant role of capelin at the mid-trophic level in the Barents Sea, causing "wasp-waist" dynamics, with capelin fluctuations strongly impacting both higher and lower trophic levels (Yaragina and Dolgov 2009).

While our results provide interesting insights into the predator-prey dynamics among zooplankton and capelin, there are some caveats worth mentioning. For example, zooplankton or other environmental factors can affect maturation not only through length, as modelled (and empirically supported, Baulier et al. 2012), but also through the condition of the capelin (Orlova et al. 2010). Furthermore, even if our model is both age-structured and accounts for some of the spatial structure in the system, there may be processes at other stages or spatial scales that can potentially affect the dynamics of the system. Similarly, species not explicitly modelled, such as competitors of capelin, could potentially influence dynamics. However, we argue that our model is a good compromise between complexity and uncertainty. With this level of complexity, we are able to tightly link the process model to available data and hence provide realistic empirically based projections of indirect ecosystem effects.

Our study shows how bottom-up effects of changes in zooplankton biomass influence capelin dynamics, and resonate back to the zooplankton community through delayed indirect effects. As capelin also plays a key role as prey of, e.g., cod, marine mammals and sea birds in the Barents Sea (Gjøsæter et al. 2009, Yaragina and Dolgov 2009), changes in zooplankton biomass may potentially cascade upwards to top-predators in the ecosystem. These results illustrate that indirect ecosystem effects such as apparent competition may play important roles in modulating ecosystem effects of environmental change. Data from the recent decade and a half indicate that the biomass of Atlantic krill species has increased in the Barents Sea, while Arctic copepod species inhabiting central and northern areas have decreased (Dalpadado et al. 2012, Johannesen et al. 2012, see also Fig. 2). Most studies focus on the physical drivers behind such changes (e.g. temperature increase, advection), but our study indicates that a krill "invasion" could additionally affect copepod 
biomass negatively through apparent competition. Specifically, our results suggest that capelin would likely benefit from a change towards increased krill dominance, and magnify it, to the disadvantage of Arctic copepods. Thus, species interactions may in this case contribute to the observed "borealization" of the Barents Sea (as found for fish species, Fossheim et al. 2015).

This study provides an example of apparent competition mediated by a shared predator, a process that may be ubiquitous in pelagic marine ecosystems but that has to date rarely been demonstrated. We propose that the sparse empirical support for apparent competition in pelagic marine ecosystems may be largely due to the difficulty in detecting it. By quantifying such indirect ecosystem effects our results and methodological approach pave the way for more realistic projections of ecosystem responses to drivers such as climate change or harvesting, in the Barents Sea as well as in other ecosystems.

\section{ACKNOWLEDGMENTS}

This study was funded by the Nordforsk-funded project Green Growth Based on Marine Resources: Ecological and Socio-Economic Constraints (GreenMAR) and by the Research Council of Norway (Projects 244647/E10 and 255487/E40). We thank Kjell Bakkeplass, Padmini Dalpadado, Gjert E. Dingsør and Elena Eriksen for help with accessing zooplankton and capelin data. Model fitting was performed on the Abel Cluster, owned by the University of Oslo and the Norwegian metacenter for High Performance Computing (NOTUR), and operated by the Department for Research Computing at USIT, the University of Oslo IT-department.

\section{Literature Cited}

Baulier, L., M. Heino, and H. Gjøsæter. 2012. Temporal stability of the maturation schedule of capelin Mallotus villosus in the Barents Sea. Aquatic Living Resources 25:151-161.

Bogstad, B., H. Gjøsæter, T. Haug, and U. Lindstrøm. 2015. A review of the battle for food in the Barents Sea: cod vs. marine mammals. Frontiers in Ecology and Evolution 3:29.

Botsford, L. W., J. C. Castilla, and C. H. Peterson. 1997. The management of fisheries and marine ecosystems. Science 277: $509-515$.

Boyce, D. G., K. T. Frank, B. Worm, and W. C. Leggett. 2015. Spatial patterns and predictors of trophic control in marine ecosystems. Ecology Letters 18:1001-1011.

Buren, A. D., M. Koen-Alonso, P. Pepin, F. Mowbray, B. Nakashima, G. Stenson, N. Ollerhead, and W. A. Montevecchi. 2014. Bottom-up regulation of capelin, a keystone forage species. PLoS ONE 9:e87589.

Casini, M., J. Hjelm, J.-C. Mollinero, J. Lövgren, M. Cardinale, V. Bartolino, A. Belgrano, and G. Kornilovs. 2009. Trophic cascades promote threshold-like shifts in pelagic marine ecosystems. Proceedings of the National Academy of Sciences of the United States of America 106:197-202.

Chaneton, E. J., and M. B. Bonsall. 2000. Enemy-mediated apparent competition: empirical patterns and the evidence. Oikos 88:380-394.

Clark, J. S., and O. N. Bjørnstad. 2004. Population time series: process variability, observation errors, missing values, lags, and hidden states. Ecology 85:3140-3150.

Collie, J. S., L. W. Botsford, A. Hastings, I. C. Kaplan, J. L. Largier, P. A. Livingston, É. Plagányi, K. A. Rose, B. K. Wells, and F. E. Werner. 2016. Ecosystem models for fisheries management: finding the sweet spot. Fish and Fisheries 17:101-125.

Dalpadado, P., and F. Mowbray. 2013. Comparative analysis of feeding ecology of capelin from two shelf ecosystems, off Newfoundland and in the Barents Sea. Progress in Oceanography 114:97-105.

Dalpadado, P., B. Bogstad, H. Gjøsæter, S. Mehl, and H. R. Skjoldal. 2002. Zooplankton-fish interactions in the Barents Sea. Pages 269-291 in K. Sherman and H. R. Skjoldal, editors. Large marine ecosystems of the North Atlantic. Elsevier, Amsterdam, The Netherlands.

Dalpadado, P., A. Yamaguchi, B. Ellertsen, and S. Johannessen. 2008. Trophic interactions of macro-zooplankton (krill and amphipods) in the Marginal Ice Zone of the Barents Sea. Deep-Sea Research 55:2266-2274.

Dalpadado, P., R. B. Ingvaldsen, L. C. Stige, B. Bogstad, T. Knutsen, G. Ottersen, and B. Ellertsen. 2012. Climate effects on the Barents Sea ecosystem dynamics. ICES Journal of Marine Science 69:1303-1316.

Dalpadado, P., K. R. Arrigo, S. S. Hjøllo, F. Rey, R. B. Ingvaldsen, E. Sperfeld, G. L. van Dijken, L. C. Stige, A. Olsen, and G. Ottersen. 2014. Productivity in the Barents Sea - Response to recent climate variability. PLoS ONE 9:e95273.

DeCesare, N. J., M. Hebblewhite, H. S. Robinson, and M. Musiani. 2010. Endangered, apparently: the role of apparent competition in endangered species conservation. Animal Conservation 13:353-362.

Eriksen, E., and P. Dalpadado. 2011. Long-term changes in krill biomass and distribution in the Barents Sea: are the changes mainly related to capelin stock size and temperature conditions? Polar Biology 34:1399-1409.

Eriksen, E., P. Dalpadado, and A. Dolgov. 2016a. Chapter 4.3.3. Biomass indices and distribution of krill and amphipods. Pages 27-29 in D. Prozorkevich and K. Sunnanå, editors. Survey report from the joint Norwegian/Russian ecosystem survey in the Barents Sea and adjacent waters, August - October 2015. IMR/PINRO Joint Report Series, No. 1/2016, 147 pp. ISSN 1502-8828. Institute of Marine Research, Bergen, Norway.

Eriksen, E., H. R. Skjoldal, A. V. Dolgov, P. Dalpadado, E. L. Orlova, and D. V. Prozorkevich. 2016b. The Barents Sea euphausiids: methodological aspects of monitoring and estimation of abundance and biomass. ICES Journal of Marine Science 73:1533-1544.

Eriksen, E., H. R. Skjoldal, H. Gjøsæter, and R. Primicerio, 2017. Spatial and temporal changes in the Barents Sea pelagic compartment during the recent warming. Progress in Oceanography 151:206-226.

Fauchald, P., M. Mauritzen, and H. Gjøsæter. 2006. Densitydependent migratory waves in the marine pelagic ecosystem. Ecology 87:2915-2924.

Fossheim, M., R. Primicerio, E. Johannesen, R. B. Ingvaldsen, M. M. Aschan, and A. V. Dolgov. 2015. Recent warming leads to a rapid borealization of fish communities in the Arctic. Nature Climate Change 5:673-677.

Frank, K. T., B. D. Petrie, J. S. Choi, and W. C. Leggett. 2005. Trophic cascades in a formerly cod-dominated ecosystem. Science 308:1621-1623.

Frank, K. T., B. D. Petrie, N. L. Shackell, and J. S. Choi. 2006. Reconciling differences in trophic control in mid-latitude ecosystems. Ecology Letters 9:1096-1105.

Frid, A., and J. Marliave. 2010. Predatory fishes affect trophic cascades and apparent competition in temperate reefs. Biology Letters 6:533-536.

Gelman, A., and D. B. Rubin. 1992. Inference from iterative simulation using multiple sequences. Statistical Science 7: $457-472$. 
Gjøsæter, H. 1998. The population biology and exploitation of capelin (Mallotus villosus) in the Barents Sea. Sarsia 83:453-496.

Gjøsæter, H., and B. Bogstad. 1998. Effects of the presence of herring (Clupea harengus) on the stock-recruitment relationship of Barents Sea capelin (Mallotus villosus). Fisheries Research 38:57-71.

Gjøsæter, H., and H. Loeng. 1987. Growth of the Barents Sea capelin, Mallotus villosus, in relation to climate. Environmental Biology of Fishes 20:293-300.

Gjøsæter, H., P. Dalpadado, and A. Hassel. 2002. Growth of Barents Sea capelin (Mallotus villosus) in relation to zooplankton abundance. ICES Journal of Marine Science 59:959-967.

Gjøsæter, H., B. Bogstad, and S. Tjelmeland. 2009. Ecosystem effects of the three capelin stock collapses in the Barents Sea. Marine Biology Research 5:40-53.

Hassel, A., H. R. Skjoldal, H. Gjøsæter, H. Loeng, and L. Omli. 1991. Impact of grazing from capelin (Mallotus villosus) on zooplankton: a case-study in the northern Barents Sea in August 1985. Polar Research 10:371-388.

Hjermann, D. Ø., G. Ottersen, and N. C. Stenseth. 2004. Competition among fishermen and fish causes the collapse of Barents Sea capelin. Proceedings of the National Academy of Sciences of the United States of America 101:11679-11684.

Hjermann, D. Ø., B. Bogstad, G. E. Dingsør, H. Gjøsæter, G. Ottersen, A. M. Eikeset, and N. C. Stenseth. 2010. Trophic interactions affecting a key ecosystem component: a multistage analysis of the recruitment of the Barents Sea capelin (Mallotus villosus). Canadian Journal of Fisheries and Aquatic Sciences 67:1363-1375.

Holt, R. D. 1977. Predation, apparent competition, and the structure of prey communities. Theoretical Population Biology 12:197-229.

ICES. 2016. Report of the Arctic fisheries working group (AFWG), 19-25 April 2016, ICES HQ, Copenhagen, Denmark. ICES CM 2016/ACOM:06.

Ives, A. R., B. Dennis, K. L. Cottingham, and S. R. Carpenter. 2003. Estimating community stability and ecological interactions from time-series data. Ecological Monographs 73:301-330.

Johannesen, E., R. B. Ingvaldsen, B. Bogstad, P. Dalpadado, E. Eriksen, H. Gjøsæter, T. Knutsen, M. Skern-Mauritzen, and J. E. Stiansen. 2012. Changes in Barents Sea ecosystem state, 1970-2009: climate fluctuations, human impact, and trophic interactions. ICES Journal of Marine Science 69:880-889.

Langangen, Ø., J. Ohlberger, L. C. Stige, J. M. Durant, E. Ravagnan, N. C. Stenseth, and D. Ø. Hjermann. 2017. Cascading effects of mass mortality events in Arctic marine communities. Global Change Biology 23:283-292.

Lynam, C. P., M. Llope, C. Mollmann, P. Helaoutet, G. A. Bayliss-Brown, and N. C. Stenseth. 2017. Interaction between top-down and bottom-up control in marine food webs. Proceedings of the National Academy of Sciences of the United States of America 114:1952-1957.

May, R. M. 1974. Stability and complexity in model ecosystems. Second edition.. Princeton University Press, Princeton, New Jersey, USA.

May, R. M., J. R. Beddington, C. W. Clark, S. J. Holt, and R. M. Laws. 1979. Management of Multispecies Fisheries. Science 205:267-277.
Monstad, T. 1971. Alder, vekst og utbredelse av lodde (Mallotus villosus) i Barentshavet og ved kysten av Nord-Norge 19681970. (Age, growth and geographical distribution of capelin (Mallotus villosus) in the Barents Sea and at the coast of northern Norway 1968-70). Cand. real. Thesis. Department of Marine Biology, University of Bergen, Bergen, Norway.

Ockendon, N., et al. 2014. Mechanisms underpinning climatic impacts on natural populations: altered species interactions are more important than direct effects. Global Change Biology 20:2221-2229.

Orlova, E. L., A. V. Dolgov, G. B. Rudneva, I. A. Oganin, and L. L. Konstantinova. 2009. Trophic relations of capelin Mallotus villosus and polar cod Boreogadus saida in the Barents Sea as a factor of impact on the ecosystem. Deep-Sea Research II 56:2054-2067.

Orlova, E. L., G. B. Rudneva, P. E. Renaud, K. Eiane, V. Savinov, and A. S. Yurko. 2010. Climate impacts on feeding and condition of capelin Mallotus villosus in the Barents Sea: evidence and mechanisms from a 30 year data set. Aquatic Biology 10:105-118.

Orlova, E. L., P. Dalpadado, T. Knutsen, V. N. Nesterova, and I. P. Prokopchuk. 2011. Zooplankton. Pages 90-119 in T. Jakobsen and V. K. Ozhigin, editors. The Barents Sea. Ecosystem, resources, management. Tapir Academic Press, Trondheim, Norway.

Pedersen, T., and M. Fossheim. 2008. Diet of 0-group stages of capelin (Mallotus villosus), herring (Clupea harengus) and cod (Gadus morhua) during spring and summer in the Barents Sea. Marine Biology 153:1037-1046.

Pikitch, E. K., et al. 2004. Ecosystem-Based Fishery Management. Science 305:346-347.

Plagányi, É. E., et al. 2014. Multispecies fisheries management and conservation: tactical applications using models of intermediate complexity. Fish and Fisheries 15:1-22.

Plummer, M. 2016. rjags: Bayesian graphical models using MCMC. R package version 4-6. https://CRAN.R-project.org/ package $=$ rjags

Skjoldal, H. R., H. Gjøsæter, and H. Loeng. 1992. The Barents Sea ecosystem in the 1980s: ocean climate, plankton, and capelin growth. ICES Marine Science Symposium 195:278-290.

Solymos, P. 2010. dclone: Data cloning in R. R Journal 2:29-37.

Søreide, J. E., E. Leu, J. Berge, M. Graeve, and S. Falk-Petersen. 2010. Timing of blooms, algal food quality and Calanus glacialis reproduction and growth in a changing Arctic. Global Change Biology 16:3154-3163.

Stige, L. C., P. Dalpadado, E. Orlova, A.-C. Boulay, J. M. Durant, G. Ottersen, and N. C. Stenseth. 2014. Spatiotemporal statistical analyses reveal predator-driven zooplankton fluctuations in the Barents Sea. Progress in Oceanography 120: 243-253.

Ushakov, N. G., and D. V. Prozorkevich. 2002. The Barents Sea capelin - a review of trophic interrelations and fisheries. ICES Journal of Marine Science 59:1046-1052.

Wooton, J. T. 1994. The nature and consequences of indirect effects in ecological communities. Annual Review of Ecology and Systematics 25:443-466.

Yaragina, N. A., and A. V. Dolgov. 2009. Ecosystem structure and resilience - A comparison between the Norwegian and the Barents Sea. Deep-Sea Research II 56:2141-2153.

\section{SUPPORTING INFORMATION}

Additional supporting information may be found in the online version of this article at http://onlinelibrary.wiley.com/doi/ 10.1002/ecy.2126/suppinfo 